\title{
A Unified Scheme of Measurement and Amplification Processes based on Micro-Macro Duality - Stern-Gerlach experiment as a typical example -
}

\author{
Ryo HARADA and Izumi OJIMA \\ Research Institute for Mathematical Sciences, \\ Kyoto University, Kyoto 606-8502, Japan
}

September 16, 2018

\begin{abstract}
A unified scheme for quantum measurement processes is formulated on the basis of Micro-Macro duality as a mathematical expression of the general idea of quantum-classical correspondence. In this formulation, we can naturally accommodate the amplification processes necessary for magnifying quantum state changes at the microscopic end of the probe system into the macroscopically visible motion of the measuring pointer. Its essence is exemplified and examined in the concrete model of the Stern-Gerlach experiment for spin measurement, where the Helgason duality controlling the Radon transform is seen to play essential roles.
\end{abstract}

\section{Introduction: Micro-Macro Duality and Mea- surements}

In this paper, we present a unified description of a measurement process of quantum observables together with the amplification process associated with it. For this purpose, we recall the essence of Micro-Macro duality [1, 2, 3] as a mathematical expression of the general idea of quantum-classical correspondence which plays crucial roles. In this context, we note that the 'boundary' between the quantum and classical levels can be found in the notion of a sector, in terms of which we can understand, in a clear-cut manner, the mutual relations between the microscopic quantum world and the macroscopic classical levels. To define a sector, we classify representations and states of a $\mathrm{C}^{*}$-algebra $\mathfrak{A}$ of quantum observables according to the quasi-equivalence $\pi_{1} \approx \pi_{2}$ [4] defined by the unitary equivalence of representations $\pi_{1}, \pi_{2}$ up to 
multiplicity, which is equivalent to the isomorphism of von Neumann algebras $\pi_{1}(\mathfrak{A})^{\prime \prime} \simeq \pi_{2}(\mathfrak{A})^{\prime \prime}$ of representatoins $\pi_{1}$ and $\pi_{2}$. A sector or a pure phase in the physical context is then defined by a quasi-equivalence class of factor representations and states corresponding to a von Neumann algebra with a trivial centre, which is a minimal unit among quasi-equivalence classes. Representations belonging to different sectors $\pi_{a}$ and $\pi_{b}$ are mutually disjoint with no non-zero intertwiners: namely, if $T$ is an intertwiner from $\pi_{a}$ to $\pi_{b}$ defined as a bounded operator $T$ from the representation space $\mathfrak{H}_{\pi_{a}}$ of $\pi_{a}$ to that $\mathfrak{H}_{\pi_{b}}$ of $\pi_{b}$ satisfying the relation $T \pi_{a}(A)=\pi_{b}(A) T(\forall A \in \mathfrak{A})$, then it vanishes, $T=0$.

If $\pi$ is not a factor representation belonging to one sector, it is called a mixed phase. In the standard situations where separable Hilbert spaces are used, a mixed phase can uniquely be decomposed into a direct sum (or integral) of sectors, through the spectral decomposition of its non-trivial centre $\mathfrak{Z}\left(\pi(\mathfrak{A})^{\prime \prime}\right)=\pi(\mathfrak{A})^{\prime \prime} \cap \pi(\mathfrak{A})^{\prime}=: \mathfrak{Z}_{\pi}(\mathfrak{A})$ of $\pi(\mathfrak{A})^{\prime \prime}$ which is a commutative von Neumann algebra admitting a 'simultaneous diagonalization'. Each sector contained in $\pi$ is faithfully parametrized by the Gel'fand spectrum $\operatorname{Spec}\left(\mathfrak{Z}_{\pi}(\mathfrak{A})\right)$ of the centre $\mathfrak{Z}_{\pi}(\mathfrak{A})$. Thus, commutative classical observables belonging to the centre physically play the role of macroscopic order parameters and the central spectrum $\operatorname{Spec}\left(\mathfrak{Z}_{\pi}(\mathfrak{A})\right)$ can be regarded as the classifying space of sectors to register faithfully all the sectors contained in $\pi$. In this way, we find in a mixed phase $\pi$ the coexistence of quantum (=intra-sectorial) and classical systems, the latter of which describes an inter-sectorial structure in terms of order parameters constituting the centre $\mathfrak{Z}_{\pi}(\mathfrak{A})$. In this way, the 'boundary' and the gap between the quantum world described by non-commutative algebras of quantum variables and the classical levels with commutative algebras of order parameters can be identified with a (superselection) sector structure consisting of a family of sectors or pure phases [1].

\section{Intra-sectorial Analysis by MASA}

Since a single sector or a pure phase corresponds to a (quasi-equivalence class of) factor representation $\pi$ of a $\mathrm{C}^{*}$-algebra $\mathfrak{A}$ of quantum observables, its intra-sectorial structure, the structure inside of a sector, is described by the observables belonging to the factor von Neumann algebra $\mathcal{M}=\pi(\mathfrak{A})^{\prime \prime}$ corresponding to $\pi$. In this and the next sections, we recapitulate the essence of the general scheme to analyze the intra-sectorial structure [2, 3]. Because of the non-commutativity of $\mathcal{M}$, what can be experimentally observed through a measurement is up to a certain maximal abelian subalgebra (MASA, for short) $\mathcal{A}=\mathcal{A}^{\prime} \cap \mathcal{M}$ (with $\mathcal{A}^{\prime}$ the commutant of $\mathcal{A}$ ) of $\mathcal{M}$ : elements of a MASA $\mathcal{A}$ can be regarded as macroscopic observables to visualize some aspects of the microscopic structure of a sector in the macroscopic form of $\operatorname{Spec}(\mathcal{A})$. In fact, a tensor product $\mathcal{M} \otimes \mathcal{A}$ (acting 
on the tensor product Hilbert space $\left.\mathfrak{H}_{\omega} \otimes L^{2}(\operatorname{Spec}(\mathcal{A}))\right)$ has a centre given by $\mathfrak{Z}(\mathcal{M} \otimes \mathcal{A})=\mathfrak{Z}(\mathcal{M}) \otimes \mathcal{A}=\mathbf{1} \otimes L^{\infty}(\operatorname{Spec}(\mathcal{A}))$, and hence, the spectrum $\operatorname{Spec}(\mathcal{A})$ of a MASA $\mathcal{A}$ to be measured can be understood as parametrizing a conditional sector structure of the composite system $\mathcal{M} \otimes \mathcal{A}$ of the observed system $\mathcal{M}$ and $\mathcal{A}$, the latter of which can be identified with the measuring apparatus $\mathcal{A}$ in the simplified version [1] of Ozawa's measurement scheme [5]. This picture of conditional sector structure is consistent with the physical essence of a measurement process as 'classicalization' of some restricted aspects $\mathcal{A}(\subset \mathcal{M})$ of a quantum system, conditional on the coupling $\mathcal{M} \otimes \mathcal{A}$ of $\mathcal{M}$ with the apparatus identified with $\mathcal{A}$.

To implement a physical process to measure the observables in $\mathcal{A}$, we need to specify a dynamical coupling between the observed and measuring systems, which is accomplished by choosing such a unitary group $\mathcal{U}$ in $\mathcal{A}$ as generating $\mathcal{A}$, i.e., $\mathcal{A}=\mathcal{U}^{\prime \prime}$. In the standard situation where the relevant Hilbert space is separable, the abelian von Neumann algebra $\mathcal{A}$ on it is generated by a single element, and hence, we can assume without loss of generality that $\mathcal{U}$ is a locally compact abelian Lie group. Because of the commutativity of $\mathcal{U}$, the group characters $\gamma$ of $\mathcal{U}, \gamma: \mathcal{U} \ni u \longmapsto \gamma(u) \in \mathbb{T}$ (: 1-dimensional torus) s.t. $\gamma\left(u_{1} u_{2}\right)=\gamma\left(u_{1}\right) \gamma\left(u_{2}\right), \gamma(e)=1$, constitute the dual goup $\widehat{\mathcal{U}}$ satisfying the Fourier-Pontryagin duality $\hat{\hat{U}} \simeq \mathcal{U}$. Since the restriction $\chi\lceil\mathcal{U}$ to $\mathcal{U} \subset \mathcal{A}$ of an algebraic character $\chi \in \operatorname{Spec}(\mathcal{A})$ of $\mathcal{A}$ is naturally a group character of $\mathcal{U}$, a canonical embedding $\operatorname{Spec}(\mathcal{A}) \hookrightarrow \widehat{\mathcal{U}}$ can be defined by $\operatorname{Spec}(\mathcal{A}) \ni \chi \longmapsto \chi \Upsilon_{\mathcal{U}} \in \widehat{\mathcal{U}}$. As the MASA $\mathcal{A}=\mathcal{A}^{\prime} \cap \mathcal{M}$ is the fixed-point subalgebra $\mathcal{A}=\mathcal{M}^{\operatorname{Ad}(\mathcal{U})}$ of $\mathcal{M}$ under the adjoint action of $\mathcal{U}$, our discussion can also be related with the Galois-theoretical context

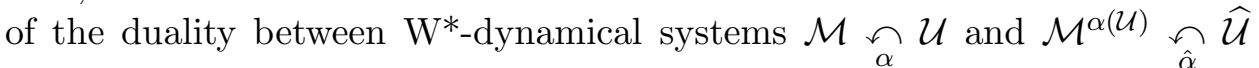
and between the associated crossed products $\mathcal{M} \rtimes_{\alpha} \mathcal{U}$ and $\mathcal{M}^{\alpha(\mathcal{U})} \rtimes_{\hat{\alpha}} \widehat{\mathcal{U}}$, where the co-action $\widehat{\alpha}$ of $\mathcal{U}$ dual to $\alpha$ can be identified with an action of $\widehat{\mathcal{U}}: \mathcal{U} \underset{\alpha}{\curvearrowright} \mathcal{M}\left(\simeq \mathcal{M}^{\alpha(\mathcal{U})} \rtimes_{\hat{\alpha}} \widehat{\mathcal{U}}\right) \rightleftarrows\left(\mathcal{M} \rtimes_{\alpha} \mathcal{U}\right)_{\hat{\alpha}} \widehat{\mathcal{U}}$. This co-action $\hat{\alpha}$ plays important roles in the reconstruction of quantum (microscopic) systems from the classical macroscopic data.

\section{Measurement Coupling, Kac-Takesaki Operators and Instrument}

We show that the above measurement coupling can be specified by means of a Kac-Takesaki operator [6] (K-T operator, for short), one of the central notions in harmonic analysis (where it is called a fundamental operator in [7] and a multiplicative unitary in [8]). In what follows this operator is seen to play essential roles in our whole scheme to unify both measurement and amplification processes. In the regular representation of the group $\mathcal{U}$, a $\mathrm{K}-\mathrm{T}$ 
operator $W$ is defined by

$$
(W \eta)(u, v):=\eta\left(v^{-1} u, v\right)
$$

for $\eta \in L^{2}(\mathcal{U} \times \mathcal{U}, d u \otimes d u), u, v \in \mathcal{U}$ with $d u$ the Haar measure of $\mathcal{U}$, characterized by the pentagonal and intertwining relations:

$$
\begin{aligned}
W_{12} W_{23} & =W_{23} W_{13} W_{12}, \\
W\left(1 \otimes \lambda_{u}\right) & =\left(\lambda_{u} \otimes \lambda_{u}\right) W,
\end{aligned}
$$

where the suffices $1,2,3$ indicate the places in the tensor product $L^{2}(\mathcal{U}, d u) \otimes$ $L^{2}(\mathcal{U}, d u) \otimes L^{2}(\mathcal{U}, d u)$ on which the operators act.

The simplest form of the action $\alpha, \mathcal{M} \curvearrowleft \mathcal{U}$, of $\mathcal{U}$ on $\mathcal{M}$ is given by the adjoint action $\alpha_{u}(M)=A d_{u}(M)=u M u^{-1}$, as commonly found in many discussions on the measurement processes. This corresponds physically to such an approximation to the coupled dynamics of the composite system $\mathcal{M} \otimes \mathcal{A}$ that the Hamiltonian $H_{0}$ intrinsic to the observed system is neglected but the bilinear coupling $H_{I}=\lambda \sum_{i} X_{i} \otimes A_{i}$ is kept between the system observables $X_{i}\left(\exp \left(i X_{i}\right) \in \mathcal{M}\right)$ and the external forces $A_{i}\left(\exp \left(i A_{i}\right) \in \mathcal{A}\right)$. To retain the effects of the dynamics intrinsic to the observed system, we take here a more general form of the action $\mathcal{M}_{\alpha} \curvearrowleft \mathcal{U}$ of the measuring system than the adjoint one under the assumption that $\alpha$ is unitarily implemented, $\alpha_{u}(M)=U_{u} M U_{u}^{-1}(M \in \mathcal{M}, u \in \mathcal{U})$, by a unitary representation $U$ of $\mathcal{U}$ on the standard representation Hilbert space $L^{2}(\mathcal{M})$ of $\mathcal{M}$. Then the representation $U(W)$ of $W$ corresponding to $\alpha=A d(U)$ is defined by

$$
(U(W) \xi)(u):=U_{u}(\xi(u)) \quad \text { for } \xi \in L^{2}(\mathcal{M}) \otimes L^{2}(\mathcal{U}, d u),
$$

satisfying the pentagonal and intertwining relations:

$$
\begin{aligned}
U(W)_{12} W_{23} & =W_{23} U(W)_{13} U(W)_{12}, \\
U(W)\left(1 \otimes \lambda_{u}\right) & =\left(U_{u} \otimes \lambda_{u}\right) U(W) .
\end{aligned}
$$

The meaning of $U(W)$ can be seen in the following heuristic expression in Dirac's bra-ket notation:

$$
U(W)=\int_{u \in \mathcal{U}} U_{u} \otimes|u\rangle d u\langle u| .
$$

This unitary operator $U(W)$ provides the coupling between the observed and measuring systems precisely required for measuring the observables in $\mathcal{A}$. For this purpose, we examine the action of its Fourier transform on the state vectors of the composite system belonging to $L^{2}(\mathcal{M}) \otimes L^{2}(\mathcal{U}, d u)$. First, in terms of the Fourier transform $(\mathcal{F} \xi)(\gamma):=\int_{\mathcal{U}} \overline{\gamma(u)} \xi(u) d u$ for $\xi \in$ $L^{2}(\mathcal{U}, d u)$, the Fourier transform $V:=(\mathcal{F} \otimes \mathcal{F}) W^{*}(\mathcal{F} \otimes \mathcal{F})^{-1}$ of the K-T operator $W$ on $L^{2}(\mathcal{U} \times \mathcal{U})$ is defined, which turns out just to be the $\mathrm{K}-\mathrm{T}$ 
operator of the dual group $\widehat{\mathcal{U}}$ (equipped with the Plancherel measure $d \hat{u}$ ) satisfying and characterized by the relations:

$$
\begin{aligned}
(V \eta)(\gamma, \chi) & =\eta\left(\gamma, \gamma^{-1} \chi\right) \quad \text { for } \eta \in L^{2}(\widehat{\mathcal{U}}, d \hat{u}), \\
V_{23} V_{12} & =V_{12} V_{13} V_{23}, \\
V\left(\lambda_{\gamma} \otimes 1\right) & =\left(\lambda_{\gamma} \otimes \lambda_{\gamma}\right) V .
\end{aligned}
$$

Similarly, the Fourier transform of $\mathcal{U}(W)$ is defined by $\widetilde{U}(V):=(i d \otimes$ $\mathcal{F}) U(W)^{*}(i d \otimes \mathcal{F})^{-1}$. Owing to the SNAG theorem due to the abelianness of $\mathcal{U}$, its unitary representation $\mathcal{U} \ni u \longmapsto U_{u} \in \mathcal{U}\left(L^{2}(\mathcal{M})\right)$ admits the spectral decomposition $U_{u}=\int_{\chi \in \operatorname{Spec}(\mathcal{A}) \subset \hat{\mathcal{U}}} \overline{\chi(u)} d E(\chi)$, corresponding to which $\widetilde{U}(V)$ has the spectral decompostion given by

$$
\widetilde{U}(V)=\int_{\chi \in \operatorname{Spec}(\mathcal{A})} d E(\chi) \otimes \lambda_{\chi}
$$

In the Dirac notation, the action of $\widetilde{U}(V)$ on $L^{2}(\mathcal{M}) \otimes L^{2}(\widehat{\mathcal{U}})$ is given for $\gamma \in \widehat{\mathcal{U}}, \xi \in L^{2}(\mathcal{M})$, by

$$
\widetilde{U}(V)(\xi \otimes|\gamma\rangle)=\int_{\chi \in \operatorname{Spec}(\mathcal{A})} d E(\chi) \xi \otimes|\chi \gamma\rangle
$$

To understand the physical meaning of the above quantities, we introduce some such vocabularies [5] as 'probe system' and 'neutral position' in measurement processes: the former means the microscopic end of the measuring apparatus at its microscopic contact point with the observed system, and the latter the initial (microscopic) state of the probe system corresponding to the macroscopically stable position of the measuring pointer realized when the apparatus is isolated.

To see clearly the essence of the formulation, we assume that $\widehat{\mathcal{U}}$ is discrete (or, equivalently, $\mathcal{U}$ is compact); then we can plug into $\gamma \in \operatorname{Spec}(\mathcal{A})(\subset \widehat{\mathcal{U}})$ and $\xi \in L^{2}(\mathcal{M})$ in Eq.(11), respectively, the group identity $\iota \in \widehat{\mathcal{U}}$ and such an eigenstate $\xi=\xi_{\chi}$ as $A \xi_{\chi}=\chi(A) \xi_{\chi}(\forall A \in \mathcal{A})$ of $\chi \in \operatorname{Spec}(\mathcal{A})$, which gives

$$
\widetilde{U}(V)\left(\xi_{\chi} \otimes|\iota\rangle\right)=\xi_{\chi} \otimes|\chi\rangle .
$$

Namely, corresponding to the eigenstate $\xi_{\chi}$ of $\mathcal{A}$ found in the observed system, the coupling unitary $\widetilde{U}(V)$ causes such a state change as $|\iota\rangle \rightarrow|\chi\rangle$ in the probe system. For such a generic state as $\xi=\sum_{\chi \in \operatorname{Spec}(\mathcal{A})} c_{\chi} \xi_{\chi}$ of the observed system, therefore, we obtain

$$
\widetilde{U}(V)(\xi \otimes|\iota\rangle)=\sum_{\chi \in \widehat{\mathcal{U}}} c_{\chi} \xi_{\chi} \otimes|\chi\rangle
$$

that is, the unitary operator $\widetilde{U}(V)$ creates from a decoupled state $\xi \otimes|\iota\rangle$ of $\mathcal{M} \otimes \mathcal{A}$ a 'perfect correlation' [9] between states of the observed system and 
of the probe system, which is just required for transmitting the information from the observed system to the probe system. When the group $\mathcal{U}$ is not compact with $\widehat{\mathcal{U}}$ not being discrete, the identity element $\iota \in \widehat{\mathcal{U}}$ is not represented by a normalized vector, $|\iota\rangle \notin L^{2}(\mathcal{U})$, but we can choose an invariant mean $m_{\mathcal{U}}$ over $\mathcal{U}$ owing to the amenability of the abelian group $\mathcal{U}$ which plays the physically equivalent roles of the neutral position $\iota$. As all what can be realized in this case is known [5] to be the approximate measurements, the formula corresponding to Eq.(3) can be given by Eq. (11) and by the use of $m_{\mathcal{U}}$ as seen below in Eq. (4). In this way the K-T operators are seen to fullfil the necessary tasks for materializing the physical essence of measurements in the mathematical formulation: the K-T operator $U(W)$ determines the coupling between the observed and the measuring systems and its Fourier transform $\widetilde{U}(V)$ given by Eq.(1) establishes the 'perfect correlation' 9].

Integrating all the ingredients relevant to our measurement scheme, we define an instrument $\mathcal{I}$ as a completely positive operation-valued measure as follows:

$$
\begin{aligned}
\mathcal{I}\left(\Delta \mid \omega_{\xi}\right)(B) & :=\left(\omega_{\xi} \otimes m_{\mathcal{U}}\right)\left(\widetilde{U}(V)^{*}\left(B \otimes \chi_{\Delta}\right) \widetilde{U}(V)\right) \\
& =\left(\left\langle\xi|\otimes\langle\iota|) \widetilde{U}(V)^{*}\left(B \otimes \chi_{\Delta}\right) \widetilde{U}(V)(|\xi\rangle \otimes|\iota\rangle)\right.\right. \\
& =\int_{\Delta} \sqrt{\frac{d E(\gamma)}{d \mu(\gamma)}} B \sqrt{\frac{d E(\gamma)}{d \mu(\gamma)}} d \mu(\gamma)=: \int_{\Delta} \sqrt{d E(\gamma)} B \sqrt{d E(\gamma)},
\end{aligned}
$$

where $\omega_{\xi}$ s.t. $\omega_{\xi}(B)=\langle\xi \mid B \xi\rangle$ is an initial state of the observed system, $d \mu(\gamma)$ an arbitrary probability measure with respect to which the spectral measure $d E(\gamma)$ of $U$ is absolutely continuous: $d E(\gamma) \ll d \mu(\gamma)$, and $\chi_{\Delta}$ the indicator function of a Borel set $\Delta \subset \operatorname{Spec}(\mathcal{A})$ to which the measured values of $\mathcal{A}$ belongs. The spectral measure $d E(\gamma)$ is just the effect of the measurement, from which our K-T operator $\widetilde{U}(V)$ can be reconstructed by $\widetilde{U}(V)=\int_{\chi \in \operatorname{Spec}(\mathcal{A})} d E(\chi) \otimes \lambda_{\chi}$. In this sense, the three notions, the K-T operator $\widetilde{U}(V)$, the effect $d E(\gamma)$ and the instrument $\mathcal{I}\left(\Delta \mid \omega_{\xi}\right)$, are all mutually equivalent. The most important essence of the statistical interpretation in the measurement processes is summarized in this notion of instrument as follows: the probability distribution for measured values of observables in $\mathcal{A}$ to be found in a Borel set $\Delta \subset \operatorname{Spec}(\mathcal{A})$ is given by $p(\Delta \mid \omega)=\mathcal{I}(\Delta \mid \omega)(\mathbf{1})$ and, associated with this, the initial state $\omega$ of the observed system is changed by the read-out of measured values in $\Delta$ into a final state given in such a form as $\mathcal{I}\left(\Delta \mid \omega_{\xi}\right) / p\left(\Delta \mid \omega_{\xi}\right)[\underline{5}$, according to which a process of the so-called 'reduction of wave packets' is described. Incidentally, the reason for the relevance of the Fourier transform from $\mathcal{U}(W)$ to $\widetilde{U}(V)=(i d \otimes \mathcal{F}) U(W)^{*}(i d \otimes \mathcal{F})^{-1}$ can naturally be understood in relation with the duality between the (algebra of) observables and the states: when the group $\mathcal{U}$ acts on the algebra $\mathcal{M}$ of the observed system, the corresponding states can be parametrized by $\widehat{\mathcal{U}}$ as "eigenstates" w.r.t. the action $U$ of $\mathcal{U}$, which should also be read out as 
the measured values.

By means of the instrument $\mathcal{I}$, a measurement process is described as the process of state changes due to the measurement coupling $\alpha=\operatorname{Ad}(U)$ which transforms an initial state $\omega$ of the observed system decoupled from the probe system into final ones of the same nature, in parallel with the scattering processes described in terms of the incoming and outgoing asymptotic states of free particles. The algebra describing the composite system is the tensor algebra $\mathcal{M} \otimes \mathcal{A}=\mathcal{M} \otimes L^{\infty}(\operatorname{Spec}(\mathcal{A}))$ realized in the initial and final stages, respectively, before and after the measuring processes according to the switching-on and -off of the coupling $\alpha=\operatorname{Ad}(U)$. As incoming and outgoing asymptotic fields, $\varphi^{\text {in }}$ and $\varphi^{\text {out }}$, in quantum field theory are interpolated by interacting Heisenberg fields $\varphi_{H}$, we can consider a similar description of the composite system of $\mathcal{M}$ and $\mathcal{A}$ with the coupled dynamics $\alpha$ incorporated at the level of the algebra which interpolates the initial and final decoupled system $\mathcal{M} \otimes \mathcal{A}$. This is given by the notion of the crossed products $\mathcal{M} \rtimes_{\alpha} \mathcal{U}$ of the algebra due to the action $\alpha$ of $\mathcal{U}$ on $\mathcal{M}$, in terms of which the effect of the measuring coupling in the measurement process can be seen in such a form as $\mathcal{M} \otimes \mathcal{A}=$ $\mathcal{M} \rtimes_{\alpha=i d_{\mathcal{M}}} \mathcal{U} \rightarrow \mathcal{M} \rtimes_{\alpha} \mathcal{U} \rightarrow \mathcal{M} \otimes \mathcal{A}$, in parallel with the scattering processes, $\varphi^{\text {in }} \rightarrow \varphi_{H} \rightarrow \varphi^{\text {out }}$. In terms of the K-T operators, the crossed product $\mathcal{M} \rtimes_{\alpha} \mathcal{U}$ as an important notion in the Fourier-Galois duality is defined on $L^{2}(\mathcal{M}) \otimes L^{2}(\mathcal{U})$ in the following two equivalent ways: either as a von Neumann algebra $\lambda^{\mathcal{M}}\left(L^{1}(\mathcal{U}, \mathcal{M})\right)^{\prime \prime}$ generated by the Fourier transform $\lambda^{\mathcal{M}}(\hat{F}):=\int_{\mathcal{U}} \hat{F}(u) U(u) d u$ of $\mathcal{M}$-valued $L^{1}$-functions $\hat{F} \in L^{1}(\mathcal{U}, \mathcal{M})$ with the convolution product, $\left(\hat{F}_{1} * \hat{F}_{2}\right)(u)=\int_{\mathcal{U}} \hat{F}_{1}(v) \alpha_{v}\left(\hat{F}_{2}\left(v^{-1} u\right)\right) d v$, mapped by $\lambda^{\mathcal{M}}$ into $\lambda^{\mathcal{M}}\left(\hat{F}_{1} * \hat{F}_{2}\right)=\lambda^{\mathcal{M}}\left(\hat{F}_{1}\right) \lambda^{\mathcal{M}}\left(\hat{F}_{2}\right)$, or, as a von Neumann algebra $\pi_{\alpha}(\mathcal{M}) \vee(1 \otimes \lambda(\mathcal{U}))$ generated by $1 \otimes \lambda(\mathcal{U})$ and by

$$
\pi_{\alpha}(\mathcal{M}):=\left\{\pi_{\alpha}(M):=\operatorname{Ad}\left(U(W)^{*}\right)(M \otimes 1) ; M \in \mathcal{M}\right\} .
$$

These two versions are related by the mapping $\alpha(W):=A d(U(W))$,

$\lambda^{\mathcal{M}}\left(L^{1}(\mathcal{U}, \mathcal{M})\right)^{\prime \prime}=(\mathcal{M} \otimes 1) \vee\left\{U_{u} \otimes \lambda_{u} ; u \in \mathcal{U}\right\} \underset{\alpha(W)}{\stackrel{\alpha(W)^{-1}}{\rightleftarrows}} \pi_{\alpha}(\mathcal{M}) \vee(1 \otimes \lambda(\mathcal{U}))$,

which can be understood as the Schrödinger and Heisenberg pictures: the former $(\mathcal{M} \otimes 1) \vee\left\{U_{u} \otimes \lambda_{u} ; u \in \mathcal{U}\right\}$ is in the Schrödinger picture with unchanged microscopic observables $\mathcal{M} \otimes 1$ and with the coupling $U_{u} \otimes \lambda_{u}$ to change macroscopic states, while, in the latter, all the coupling effects are concentrated in the observables $\pi_{\alpha}(\mathcal{M})$ in contrast to the kinematical changes of macroscopic states caused by $\lambda(\mathcal{U})$.

In the case of the instrument, the effects of the measurement coupling $\widetilde{U}(V)$ are encoded in the form of macroscopic state changes recorded in the spectrum of the non-trivial centre $\mathfrak{Z}(\mathcal{M} \otimes \mathcal{A})=\mathcal{A}=L^{\infty}(\operatorname{Spec}(\mathcal{A}))$ of 
$\mathcal{M} \otimes \mathcal{A}$, playing the same roles as the order parameters to specify sectors in the inter-sectorial context. For these reasons, the most natural physical essence of the formalism in terms of an instrument $\mathcal{I}$ can be found in the interaction picture, whose coupling term $\widetilde{U}(V)=(i d \otimes \mathcal{F}) U(W)^{*}(i d \otimes \mathcal{F})^{-1}$ is responsible for deforming the decoupled algebra $\mathcal{M} \otimes \mathcal{A}$ into the above crossed product $\mathcal{M} \rtimes_{\alpha} \mathcal{U}$.

To clarify the natural meaning of the above scheme, we note a useful analogy of the duality coupling to the familiar complementarity of DNA between $\mathrm{A}$ (denine) and $\mathrm{T}$ (hymine) and between $\mathrm{G}$ (uanine) and $\mathrm{C}$ (ytosine), repectively: the role of the coupling between $d E(\chi)$ and $\lambda_{\chi}$ in the K-T operator $\widetilde{U}(V)=\int_{\chi \in \operatorname{Spec}(\mathcal{A})} d E(\chi) \otimes \lambda_{\chi}$ is just similar to that of the complementarity of A-T and G-C, as the former implements the transcription of the data $\chi$ in the object system to the probe system in the form of $\lambda_{\chi}: \iota \rightarrow \chi$ similarly to the latter case.

At this point, we note that the above standard description of measurement processes in terms of an instrument implicitly presupposes that the quantum-theoretical processes, $\xi \rightarrow \xi_{\chi}$ and $\iota \rightarrow \chi$, taking place at the microscopic contact point of the observed and the probe systems can be directly interpreted as the measured data $\chi$ identifiable with a position of the measuring pointer visible at the macroscopic level. There exist certain mathematical and/or physical gaps between these two levels which need be filled up: to adjust theoretical descriptions to the realistic experimental situations, we need to discuss how these changes of probe systems dynamically propagate into macroscopic motions of the measuring pointer. This is just the problem of the amplification processes to amplify the invisible quantum state changes in the probe system into the macroscopic data registered in some visible form of suitable order parameters. (Continuuing the above analogy to the DNA, the aspect of amplification can naturally be compared with the process of $P C R[=$ polymer chain reaction $]$ to amplify the sequential data of DNA.) In the next section, we formulate its general and abstract essence in mathematical terms, by which the notion of the instrument need be supplemented. In view of the inevitable noises in the actual experiment situations, it is also necessary to show how the relevant information survives to reach the macroscopically visible level, which requires the estimates of the disturbance terms in the form of adiabaticity condition as will be done in 84 .

\section{Unified Description of Measurements and Am- plifications}

We note here such a remarkable property inherent in the regular representation of $\widehat{\mathcal{U}}$ as the mutual quasi-equivalence, $\lambda^{\otimes m} \approx \lambda^{\otimes n}(\forall m, n \in \mathbb{N})$, among 
its arbitrary tensor powers $\lambda^{\otimes n}:=(\widehat{\mathcal{U}} \ni \gamma \longmapsto \underbrace{\lambda_{\gamma} \otimes \cdots \otimes \lambda_{\gamma}}_{n} \in U\left(L^{2}(\widehat{\mathcal{U}})\right)^{\otimes n})$, as seen by the repeated use of the intertwining relation $V\left(\lambda_{\gamma} \otimes 1\right)=\left(\lambda_{\gamma} \otimes\right.$ $\left.\lambda_{\gamma}\right) V$ of the K-T operator $V$ :

$$
\begin{aligned}
& V_{N, N+1} \cdots V_{23} V_{12}\left(\lambda_{\gamma} \otimes 1^{\otimes N}\right) \\
& =V_{N, N+1} \cdots V_{23} V_{12}\left(\left(\lambda_{\gamma} \otimes 1\right) \otimes \cdots \otimes 1\right) \\
& =V_{N, N+1} \cdots V_{23}\left(\left(\lambda_{\gamma} \otimes \lambda_{\gamma}\right) \otimes 1 \otimes \cdots \otimes 1\right) V_{12} \\
& =V_{N, N+1} \cdots V_{23}\left(\lambda_{\gamma}^{\otimes 2} \otimes 1^{\otimes(N-1)}\right) V_{12} \\
& =\cdots=V_{N, N+1} \cdots V_{n, n+1}\left(\lambda_{\gamma}^{\otimes n} \otimes 1^{\otimes(N-n+1)}\right) V_{n-1, n} \cdots V_{23} V_{12} \\
& =\lambda_{\gamma}^{\otimes(N+1)} V_{N, N+1} \cdots V_{23} V_{12} .
\end{aligned}
$$

On this basis, we can formulate a dynamical process of amplification [10] in terms of a unitary action $T_{N}$ of $\mathbb{N}$ on the tensor algebra $\mathcal{M} \otimes\left(\otimes L^{\infty}(\widehat{\mathcal{U}})\right)$ with $\otimes L^{\infty}(\widehat{\mathcal{U}}):={\underset{N}{\longrightarrow}}_{\lim _{N}}^{L^{\infty}(\widehat{\mathcal{U}}) \otimes L^{\infty}(\widehat{\mathcal{U}}) \otimes \cdots \otimes L^{\infty}(\widehat{\mathcal{U}})}$ defined by

$$
\begin{aligned}
& T_{N}\left(A \otimes f_{2} \otimes \cdots \otimes f_{N+1}\right) \\
& :=\widetilde{U}(V)_{12}^{*} V_{23}^{*} \cdots V_{N, N+1}^{*}\left(A \otimes f_{2} \otimes \cdots \otimes f_{N+1}\right) V_{N, N+1} \cdots V_{23} \widetilde{U}(V)_{12} \\
& =A d\left(\widetilde{U}(V)_{12}^{*}\right) A d\left(V_{23}^{*}\right) \cdots A d\left(V_{N, N+1}^{*}\right)\left(A \otimes f_{2} \otimes \cdots \otimes f_{N+1}\right) \\
& =A d\left(\widetilde{U}(V)^{*}\right)\left(A \otimes A d\left(V^{*}\right)\left(f_{2} \otimes A d\left(V^{*}\right)\left(\cdots \otimes A d\left(V^{*}\right)\left(f_{N} \otimes f_{N+1}\right)\right)\right) \cdots\right) \\
& \quad \text { for } A \in \mathcal{M} \text { and } f_{i} \in L^{\infty}(\widehat{\mathcal{U}}),
\end{aligned}
$$

which is similar to the formulation of quantum Markov chain due to Accardi [11]. When $\widehat{\mathcal{U}}$ is discrete, this process can be seen in a more clear-cut way in the Schrödinger picture:

$$
\begin{aligned}
U_{N} & :=V_{N, N+1} \cdots V_{23} \widetilde{U}(V)_{12} ; \\
& U_{N}\left(\xi \otimes|\iota\rangle^{\otimes N}\right)=\sum_{\gamma \in \operatorname{Spec}(\mathcal{A})} c_{\gamma} V_{N, N+1} \cdots V_{34} V_{23}\left(\xi_{\gamma} \otimes|\gamma\rangle \otimes|\iota\rangle \otimes \cdots \otimes|\iota\rangle\right) \\
& =\sum_{\gamma \in \operatorname{Spec}(\mathcal{A})} c_{\gamma} V_{N, N+1} \cdots V_{34}\left(\xi_{\gamma} \otimes|\gamma\rangle \otimes|\gamma\rangle \otimes|\iota\rangle \otimes \cdots \otimes|\iota\rangle\right) \\
& =\cdots=\sum_{\gamma \in \operatorname{Spec}(\mathcal{A})} c_{\gamma} \xi_{\gamma} \otimes\left[|\gamma\rangle^{\otimes N}\right],
\end{aligned}
$$

where $\xi=\sum_{\chi \in \operatorname{Spec}(\mathcal{A})} c_{\chi} \xi_{\chi}$ is a generic state $\xi \in L^{2}(\mathcal{M})$ of the observed system. According to the general basic idea of 'quantum-classical correspondence', a classical macroscopic object is to be identified with a condensed state of infinite number of quanta, as well exemplified by the macroscopic magnetization of Ising or Heisenberg ferromagnets described by the aligned states $|\uparrow\rangle^{\otimes N}$ of 'infinite number' $N \gg 1$ of microscopic spins. Likewise, the 
states $|\iota\rangle^{\otimes N}:=\underbrace{|\iota\rangle \otimes|\iota\rangle \otimes \cdots \otimes|\iota\rangle}_{N}$ and $|\gamma\rangle^{\otimes N}:=\underbrace{|\gamma\rangle \otimes|\gamma\rangle \otimes \cdots \otimes|\gamma\rangle}_{N}$ (with $N \gg 1$ ) can physically be interpreted as representing macroscopic positions of the measuring pointer corresponding, respectively, to the initial and final probe states parametrized by $\iota$ and $\gamma$. Thus the above repeated action $V_{N, N+1} \cdots V_{23} \widetilde{U}(V)_{12}$ of the K-T operator $V$ describes a cascade process or a domino effect of 'decoherence', which, triggered by the initial data $\xi_{\gamma}$ of the observed system, amplifies a probe state change $|\iota\rangle \rightarrow|\gamma\rangle$ at the microscopic end of the apparatus to be transferred into the macroscopic classical motion $\iota \rightarrow \gamma$ of the measuring pointer.

In view of the above aspects, we define a unified version of the instrument combined with the amplification process:

$$
\widehat{\mathcal{I}}_{N}\left(\Delta \mid \omega_{\xi}\right)=\left(\omega_{\xi} \otimes m_{\mathcal{U}}^{\otimes N}\right)\left(U_{N}^{*}\left((-) \otimes \chi_{\Delta}^{\otimes N}\right) U_{N}\right),
$$

in terms of which we can give an affirmative answer to the question posed at the end of the previous section, $\S 2$, concerning the realistic meaning of the quantity $\Delta$ as the actual data to be read out from the measuring pointer. To this end, we show the equality

$$
\mathcal{I}\left(\Delta \mid \omega_{\xi}\right)=\widehat{\mathcal{I}}_{N}\left(\Delta \mid \omega_{\xi}\right)
$$

between the usual and the above instruments as follows: assuming the discreteness of $\widehat{\mathcal{U}}$ for simplicity, we calculate for $B \in \mathcal{M}$,

$$
\begin{aligned}
& \widehat{\mathcal{I}}_{N}\left(\Delta \mid \omega_{\xi}\right)(B)=\left(\omega_{\xi} \otimes m_{\mathcal{U}}{ }^{\otimes N}\right)\left(U_{N}^{*}\left(B \otimes \chi_{\Delta}^{\otimes N}\right) U_{N}\right) \\
& =\left(\sum _ { \chi _ { 1 } \in \operatorname { S p e c } ( \mathcal { A } ) } c _ { \chi _ { 1 } } ^ { * } \left\langle\xi _ { \chi _ { 1 } } \left|\otimes\left\langle\left.\chi_{1}\right|^{\otimes N}\right)\left(B \otimes \chi_{\Delta}^{\otimes N}\right)\left(\sum_{\chi_{2} \in \operatorname{Spec}(\mathcal{A})} c_{\chi_{2}}\left|\xi_{\chi_{2}}\right\rangle \otimes\left|\chi_{2}\right\rangle^{\otimes N}\right)\right.\right.\right. \\
& =\sum_{\chi \in \Delta}\left|c_{\chi}\right|^{2}\left\langle\xi_{\chi}|B| \xi_{\chi}\right\rangle \chi_{\Delta}(\chi)^{N}=\sum_{\chi \in \Delta}\left|c_{\chi}\right|^{2}\left\langle\xi_{\chi}|B| \xi_{\chi}\right\rangle \\
& =\mathcal{I}\left(\Delta \mid \omega_{\xi}\right)(B)
\end{aligned}
$$

which reduces for $\Delta=\{\gamma\}(\subset \operatorname{Spec}(\mathcal{A}))$ to such a familiar result as

$$
\begin{aligned}
\mathcal{I}\left(\{\gamma\} \mid \omega_{\xi}\right)(B) & =\widehat{\mathcal{I}}_{N}\left(\{\gamma\} \mid \omega_{\xi}\right)(B)=\left|c_{\gamma}\right|^{2}\left\langle\xi_{\gamma}|B| \xi_{\gamma}\right\rangle ; \\
p\left(\{\gamma\} \mid \omega_{\xi}\right) & =\mathcal{I}\left(\{\gamma\} \mid \omega_{\xi}\right)(\mathbf{1})=\widehat{\mathcal{I}}_{N}\left(\{\gamma\} \mid \omega_{\xi}\right)(\mathbf{1}) \\
& =\left|c_{\gamma}\right|^{2} \text { for } \forall N \in \mathbb{N}, \gamma \in \operatorname{Spec}(\mathcal{A}) .
\end{aligned}
$$

Since $\left|c_{\gamma}\right|^{2}$ gives precisely the probability of finding a macroscopic state $|\gamma\rangle^{\otimes N}$, we have observed just the agreement of the probability distributions between the one arising from the microscopic system-probe coupling and the final result realized through the amplification process. This fact ensures the pertinence of instruments for the description of measurements, giving a clear-cut version of quantum-classical correspondence. 


\section{Infinite divisibility and Lévy process}

The unitarity of the above amplification process is guaranteed by the quasiequivalence relations among arbitrary tensor powers $\lambda^{\otimes n}=\lambda \otimes \cdots \otimes \lambda$ of the regular representation $\lambda$ of $\widehat{\mathcal{U}}$. It can also explain the possibility of the recurrent quantum interference even after the contact of a quantum system with the measuring apparatus when the number $N$ of repetition need not be regarded as a real infinity. This point is evident from Eq.(5) which is valid independently of $N \in \mathbb{N}$. In general, the problem as to whether the situation is made 'completely' classical or not depends highly on the relative configurations among many large or small numbers, which can consistently be described in the framework of the non-standard analysis (see, for instance, [12]).

In close relation to this, it is also interesting to note that the above amplification process is related to a Lévy process through its 'infinite divisibility' as follows: similarly to the affine property $f(\lambda x+\mu y)=\lambda f(x)+$ $\mu f(y)(\forall \lambda, \mu>0)$ of a map $f$ defined on a convex set following from the additivity $f(x+y)=f(x)+f(y)$, we can extrapolate the relation $\lambda^{m} \approx \lambda^{n}(\forall m, n \in \mathbb{N})$ into $\lambda \approx \lambda^{n / m}$, which means the infinite divisibility $(A d(V))^{t+s} \approx(A d(V))^{t}(A d(V))^{s}(t, s>0)$ of the process induced by the above transformation. In this way, we see that simple individual measurements with definite measured values are connected without gaps with discrete and/or continuous repetitions of measurements [13. If this formulation exhausts the essence of the problem, the remaining tasks reduce to its physical and/or technical implementation through suitable choices of the media connecting the microscopic contact point between the system and the apparatus to the measuring pointer. In such contexts, we need to examine some aspects concerning the stability of the information transmitted from microscopic to macroscopic levels, as will be seen in the next section.

\section{Example Case of Stern-Gerlach Experiment}

In this section we apply the scheme developed so far to the experimental situation of Stern-Gerlach type to check the validity of its general essence and to attain a deeper understanding of it through the concrete example. We will find also the necessity of some generalization or modification for adapting the scheme to actual situations. The essence of Stern-Gerlach experiment:1] [14, 15. can be found in the coupling between the (spin and/or orbital) angular momentum of the quantum particles (such as atoms or electrons) and the inhomogeneous external magnetic field, according to which the microscopic differences in the quantized directions of angular momentum are amplified into the macroscopic distance of the arriving points of the particle. For

\footnotetext{
${ }^{1}$ Suggested by O.Stern \& W.Gerlach in 1922
} 
simplicity, we consider here the spin $\boldsymbol{\sigma}=\left(\sigma_{x}, \sigma_{y}, \sigma_{z}\right)$ of an electron (with spin $s=1 / 2$ ), whose associated magnetic moment $\mu \boldsymbol{\sigma}$ couples to the magnetic field via the interaction term $\mu \boldsymbol{\sigma} \cdot \mathbf{B}(\mathbf{x})$ : through the $\mathbf{x}$-dependence of $\mathbf{B}(\mathbf{x})$ due to its inhomogeneity, this coupling causes the orbital change of the electron according to its spin direction (up or down) with respect to the defined axis (see Fig. 1 in 4.1 ). Thus the magnetic field $\mathbf{B}(\mathbf{x})$ is seen to play a double role; the coupling $\mu \boldsymbol{\sigma} \cdot \mathbf{B}$ causes, on the one hand, the spectral decomposition of the quantum spin $\boldsymbol{\sigma}$, and it causes, on the other hand, the amplification process through its dependence on $\mathbf{x}$. Through the process, we can 'see' the quantum spin variable of the electron as the separation of its spatial orbits (or, more directly, the arriving points on the screen). Thus the two states $|\uparrow\rangle$ and $|\downarrow\rangle$, respectively, of spin up and down, can be distinguished through the amplification process caused by the Stern-Gerlach measurement apparatus.

In 93 the amplification process was formulated in its idealized abstract form in terms of the homogeneous repetition by a K-T operator. In the present case of Stern-Gerlach experiment, however, the coupling between the electron and the inhomogeneous magnetic field depends on the position of the moving electron, owing to which the unitary coupling term $V=\exp \left[\frac{i t}{\hbar} \mu \boldsymbol{\sigma} \otimes \mathbf{B}(\mathbf{x})\right]$ depends on the position $\mathbf{x}$ of the electron along its trajectory. At the same time, any amplification processes cannot get rid of noise effects to disturb the ideal separations between upward and downward electron beams corresponding to macroscopically distinguishable states $|\uparrow\rangle^{\otimes \infty}$ and $|\downarrow\rangle^{\otimes \infty}$, respectively. For these reasons, it is necessary to examine whether the possible spin-flips during the travel of electron through the magnetic field can sufficiently be suppressed. Otherwise, frequent spin-flips may destroy the meaningful connection between the spin variables of the electrons and the points on the screen to detect them. Therefore, to ensure the distinguishability and the stability in the separations of final results, some physical conditions need be supplemented to ensure that these 'error probability' is small enough. This can be understood as a kind of 'adiabaticity condition' related with the validity of adiabatic approximation to treat the varying and fluctuating background field.

\subsection{Applying the scheme to Stern-Gerlach experiment}

The standard setting of the Stern-Gerlach experiment is shown below (see Fig.1 to illustrate the apparatus); we prepare a given type of metal which emits the electron beam through the thermal oscillation. The thermal electronic beam enters in the inhomogeneous magnetic field $\mathbf{B}(\mathbf{x})$ generated between magnetic poles which covers a spatial region with a length scale of the order of a meter. The orbital motion of each electron is bent upward or downward according to the directions of its spin coupled to the magnetic field; the microscopic state determined by the direction of electron spin as 
an invisible internal degree of freedom is thus converted into the visible macroscopic form of spatial separations of the spots on the screen caused by the electrons.

From here, we focus on the situation for detecting the spin direction consisting of an electron with spin $s=1 / 2$, mass $m$, charge $e$, magnetic moment $\mu\left(=e \hbar / 2 \mu_{0} m c\right.$ with magnetic permeability $\mu_{0}$ of vacuum $)$ and of the external magnetic field whose direction is supposed to be fixed in the $z$-axis.

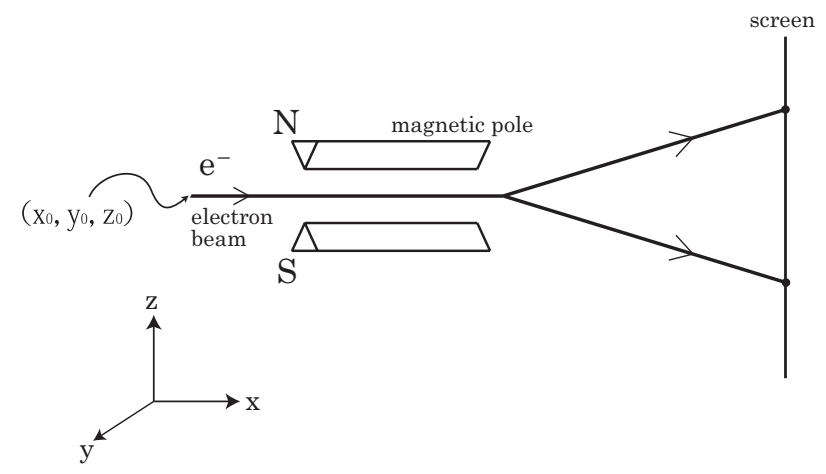

Figure 1: The settings of Stern-Gerlach experiment with single pair of magnetic poles

For applying our general scheme, we should proceed in the following steps:

0) To find the algebra which describes the physical system.

1) To extract the basic ingredients relevant to Micro-Macro duality (MASA, unitary group and their duals) from the algebra found in 0 ).

2) To identify the K-T operator in terms of these ingredients.

0) To find the algebra which describes the physical system. The physical variables of the electron constitute the algebra $M_{2}(\mathbb{C}) \otimes B\left(L^{2}\left(\mathbb{R}^{3}\right)\right)$ consisting of the spin variables $M_{2}(\mathbb{C})=\operatorname{Lin}\left(\sigma_{x}, \sigma_{y}, \sigma_{z}\right)^{\prime \prime}$ and the algebra $B\left(L^{2}\left(\mathbb{R}^{3}\right)\right)$ of the canonical commutation relations (a CCR algebra, for short, or, a Heisenberg algebra) generated, respectively, by Pauli matrices $\sigma_{i}$ and by the spatial coordinates $x, y, z$ and the momenta $p_{x}, p_{y}, p_{z}$. According to the general framework in $₫ \mathbb{1}$, we can take $\mathcal{M}=M_{2}(\mathbb{C}) \otimes B\left(L^{2}\left(\mathbb{R}^{3}\right)\right)$ as the algebra describing the system to be observed (as a von Neumann algebra of type I). 
1) To extract basic ingredients relevant to Micro-Macro duality. We can find the MASA as

$$
\mathcal{A}=\mathcal{A}^{\prime}=\operatorname{Diag}(2, \mathbb{C}) \otimes L^{\infty}\left(\mathbb{R}^{3}\right),
$$

up to unitary conjugacy, where $\operatorname{Diag}(2, \mathbb{C})$ denotes the set of $2 \times 2$ diagonal matrices $\left(\begin{array}{ll}\alpha & 0 \\ 0 & \beta\end{array}\right)(\alpha, \beta \in \mathbb{C})$. This algebra is generated by the group $\mathcal{U}=\mathcal{U}(\mathcal{A})$ of its unitary elements:

$$
\mathcal{U}=\mathcal{U}(\mathcal{A})=\mathbb{T}^{2} \otimes \mathcal{U}\left(L^{\infty}\left(\mathbb{R}^{3}\right)\right)=\mathbb{T}^{2} \otimes L^{\infty}\left(\mathbb{R}^{3}, \mathbb{T}\right) .
$$

The dual objects are also determined as follows:

Spectrum: $\operatorname{Spec}(\mathcal{A})=\{ \pm 1\} \times \mathbb{R}^{3}$;

Dual group: $\widehat{\mathcal{U}}=\mathbb{Z}^{2} \otimes L^{1}\left(\mathbb{R}^{3}, \mathbb{Z}\right)$,

where $L^{1}\left(\mathbb{R}^{3}, \mathbb{Z}\right)$ consists of compactly supported $\mathbb{Z}$-valued step functions on $\mathbb{R}^{3}$, namely, each element $f \in L^{1}\left(\mathbb{R}^{3}, \mathbb{Z}\right)$ takes a constant integer value $c_{i} \in \mathbb{Z}$ on each $\Delta_{i}$ of a finite number of non-intersecting Borel sets $\Delta_{1}, \cdots, \Delta_{r}$ in $\mathbb{R}^{3}$ and vanishes outside of $\bigcup_{i=1, \cdots, r} \Delta_{i}: f(x)=\left\{\begin{array}{l}c_{i} \text { for } x \in \Delta_{i}, \\ 0 \text { otherwise. }\end{array}\right.$

We note that it is possible to extract the information on the spin degrees of freedom of the observed system from the spin algebra only, ignoring the orbital part described by the CCR. In this context, the relevant MASA $\left\{\sigma_{z}\right\}^{\prime \prime}$ is just the Cartan subalgebra of the Lie algebra $\mathfrak{s u}(2)=\operatorname{Lin}\left(\sigma_{x}, \sigma_{y}, \sigma_{z}\right)$ (as is familiar in the theory of semi-simple Lie algebras), where the spectrum $\mathbb{Z}_{2}=\{ \pm 1\} \subset \operatorname{Spec}(\mathcal{A})$ can be identified with its root system. Physically they correspond to the spin up/down states with respect to the $z$-axis. In contrast to $\operatorname{Spec}(\mathcal{A})$ having no identity element in itself, we can identify the unit element $(0,0) \in \mathbb{Z}^{2}$ of the dual group $\mathbb{Z}^{2}$ as the neutral position of the measuring system, which can also be identified with the Haar measure $d t_{1} d t_{2}$ of $\mathbb{T}^{2}$ or the constant function 1 on $\mathbb{T}^{2}$. While this neutral position does not exist as a position of measuring pointer, operationally it represents a situation of no click on either of upper or lower detector. Generic states of electron spin to be measured are represented by arbitrary superpositions $c_{+}|\uparrow\rangle+c_{-}|\downarrow\rangle\left(c_{+}, c_{-} \in \mathbb{C},\left|c_{+}\right|^{2}+\left|c_{-}\right|^{2}=1\right)$ of two eigenstates $|\uparrow\rangle,|\downarrow\rangle$ of $\sigma_{z}$. According to the result in $\$ 2$, the coefficient $c_{ \pm}$gives the transition amplitude from the above 'state' of neutral position (of the measuring pointer) to either of the 'amplified' macroscopic states $|\uparrow\rangle^{\otimes N}$ and $|\downarrow\rangle{ }^{\otimes N}$.

2) To identify the K-T operator. Our aim here is to understand the role of the coupling Hamiltonian $\mu \boldsymbol{\sigma} \otimes \mathbf{B}(\mathbf{x})$ in relation with a K-T operator and its associated instrument. For this purpose, we consider a (trivial) vector 
bundle $E:=\mathbb{R}^{3} \times \mathbb{C}^{2} \rightarrow \mathbb{R}^{3}$ over a base space $\mathbb{R}^{3}$ spanned by the electron coordinates $\mathbf{x}$ with a fibre $\mathbb{C}^{2} \ni \psi(\mathbf{x})$ describing spin states of the electron at $\mathbf{x} ; E$ has group actions on its base space and its standard fibre, respectively, by the 3 -dimensional motion group $M(3)=\mathbb{R}^{3} \underset{A d}{\rtimes} S U(2)=: G$ and by the spin rotations $S U(2)=: H$, where $\rtimes$ means the semi-direct product w.r.t. the adjoint action of $S U(2)$ on $\mathbb{R}^{3} \simeq\left\{X \in M_{2}(\mathbb{C}) ; \operatorname{Tr}(X)=0, X^{*}=\right.$ $X\}$. It is important here to note that $E$ is a homogeneous bundle over the homogeneous space $G / H \simeq \mathbb{R}^{3}$, according to which a representation of $G$ can be induced from that of its subgroup $H$. Therefore, the geometry involved in the Stern-Gerlach experiment (as an intra-sectorial version) can be related to the measurement scheme [1, 16] for a sector bundle $G \underset{H}{\times} \hat{H} \rightarrow G / H$ over $G / H$ consisting of the degenerate vacua associated to a spontaneous symmetry breaking of $G$ into an unbroken subgroup $H$ with the standard fibre $\hat{H}$ describing the sector structure associated with $H$ :

$$
\left[\begin{array}{ccc}
\mathcal{M}^{H} \rtimes \widehat{G} \simeq \mathcal{M} \rtimes \widehat{(H \backslash G)} & \Longrightarrow & \begin{array}{l}
\text { read-out data in Spec(centre) } \\
\text { (I) }=G / H: \text { degenerate vacua }
\end{array} \\
\Uparrow \hat{G}^{\Uparrow}\left[\mathcal{M} \rtimes H \simeq \mathcal{M}^{H}\right] & \Longrightarrow & \text { read-out data in Spec(centre) } \\
: \text { coupling (I) } & (\mathrm{II})=\hat{H} \text { : sectors on a vacuum } \\
\text { coupling (II): } H \curvearrowright \mathcal{M} &
\end{array}\right]
$$

The interpretation of each step of (I) and (II) in this diagram is just in parallel with our measurement scheme: the unbroken subgroup $H$ acts on the algebra $\mathcal{M}$ of observables of the system through the coupling (II), according to which the associated sector structure over a fixed vacuum can be read off (II) in terms of $\hat{H}$ realized as the spectrum of the centre of $\mathcal{M} \rtimes H$, and, similarly, the coupling (I) to implement the co-action of $G$ on the crossed product $\mathcal{M} \rtimes H$ makes it possible to observe the sector structure (I) of the degenerate vacua parametrized by $G / H$. From this viewpoint, the interaction Hamiltonian $\boldsymbol{\sigma} \otimes \mu \mathbf{B}(\mathbf{x})=\sigma_{z} \otimes \mu B_{z}(\mathbf{x})=\left(\begin{array}{cc}\mu B_{z}(\mathbf{x}) & 0 \\ 0 & -\mu B_{z}(\mathbf{x})\end{array}\right)$ can be interpreted as follows: the coupling term $\exp \left[\frac{i t}{\hbar} \sigma_{z} \otimes \mu B_{z}\right]$ exhibits, via spectral decomposition, the 'sector' structure $\sigma_{z}= \pm 1$ parametrized by the roots \pm 1 of $H=S U(2)$ similarly to the above (II) within a fibre. When we recall the $\mathbf{x}$-dependence of $B_{z}=B_{z}(\mathbf{x}), \mathbf{x} \in \mathbb{R}^{3}=G / H$, the aspects (I) of the degenerate vacua as condensed states shows up in relation with the base space $G / H=\mathbb{R}^{3}$. To see this, we consider such an approximation of the inhomogeneus magnetic field $B_{z}(\mathbf{x})$ as

$$
B_{z}(\mathbf{x}) \simeq B_{0}+\frac{\partial B_{z}}{\partial z} z=B_{0}+B_{1} z
$$


This allows us to interpret the above coupling term $\exp \left[\frac{i}{\hbar} \Delta t \sigma_{z} \otimes \mu B_{z}\right]$ (for a time interval $\Delta t$ ) as another K-T operator relevant to (I):

$$
\begin{aligned}
\exp \left[\frac{i}{\hbar} \Delta t \sigma_{z} \otimes \mu B_{z}(\mathbf{x})\right] & =\left(\begin{array}{cc}
\exp \left[\frac{i}{\hbar} \mu B_{z}(\mathbf{x}) \Delta t\right] & 0 \\
0 & \exp \left[-\frac{i}{\hbar} \mu B_{z}(\mathbf{x}) \Delta t\right]
\end{array}\right) \\
& \simeq e^{\frac{i}{\hbar} \sigma_{z} \mu B_{0} \Delta t}\left(\begin{array}{cc}
e^{\frac{i}{\hbar} \mu B_{1} z \Delta t} & 0 \\
0 & e^{-\frac{i}{\hbar} \mu B_{1} z \Delta t}
\end{array}\right),
\end{aligned}
$$

which describes the (co-)action of the $z$-axis $\mathbb{R} \subset G / H$ on $\mathcal{M}$ to generate $\mathcal{M} \rtimes \widehat{(H \backslash G)}$ (an augmented algebra introduced in 1]). To understand this, it is sufficient to note that the exponent $\pm \frac{i}{\hbar} \mu B_{1} z \Delta t$ of matrix elements $\exp \left( \pm \frac{i}{\hbar} \mu B_{1} z \Delta t\right)$ in the above coupling unitary $\exp \left(\frac{i}{\hbar} \Delta t \sigma_{z} \otimes \mu B_{z}\right)$ can be seen as the spectral value of the K-T operator $\exp \left(\frac{i}{\hbar} \hat{p}_{z} \otimes \hat{z}\right)=\int d E\left(p_{z}\right) \otimes \hat{\lambda}_{p_{z}}$ corresponding to the (generalized) eigenvalue $p_{z}= \pm \mu B_{1} \Delta t$ of the momentum operator $\hat{p}_{z}$ :

$$
\Delta p_{z}= \pm \mu B_{1} \Delta t \quad \text { : corresponding to the eigenvalue } \pm 1 \text { of } \sigma_{z},
$$

In the context of group representations, two representations of $G=\mathbb{R}^{3} \underset{A d}{\rtimes}$ $S U(2)$ are induced from the two representations of $H=S U(2)$ corresponding to the eigenvalues $\sigma_{z}= \pm 1$, which are restricted to another subgroup $\mathbb{R}^{3}$ and then to the $z$-axis $\subset \mathbb{R}^{3}$, corresponding to (approximately) plane waves with $p_{z}= \pm \mu B_{1} \Delta t$, which reach the upper/lower detectors, respectively:

$$
\begin{gathered}
G=\mathbb{R}^{3} \underset{A d}{\rtimes} S U(2) \\
\text { (induction : ) } \nearrow \quad \searrow(: \text { restriction) } \\
\sigma_{z} \longrightarrow \quad G / \mathbb{R}^{3}=S U(2) \underset{\text { Helgason duality }}{\longleftrightarrow} G / H=\mathbb{R}^{3} \longrightarrow \hat{p}_{z} .
\end{gathered}
$$

In this way, the spin $\boldsymbol{\sigma}$ and the orbital motion described by $\mathbf{x}, \mathbf{p}$ are coupled by the inhomogeneity of the external magnetic field $B_{z}(\mathbf{x}) \simeq B_{0}+B_{1} z$, according to which the microscopic directions $\sigma_{z}= \pm 1$ of the former is amplified into the macroscopic directions $p_{z}= \pm \mu B_{1} \Delta t$ in the orbital motion. These latter directions can be understood as the 'amplified' states, $|\uparrow\rangle^{\otimes N}$ and $|\downarrow\rangle^{\otimes N}$ with the upper/lower points on the target screen.

Comment 1 The 'Helgason duality' above is a special case of the duality between the two homogeneous spaces, $K \backslash G$ and $G / H$, constituting a double fibration $K \backslash G \nleftarrow G \rightarrow G / H$, which plays important roles in the context of Radon transforms [17].

It is remarkable that the coupling unitary $\exp \left[\frac{i t}{\hbar} \sigma_{z} \otimes \mu B_{z}(\mathbf{x})\right]$ characteristic of the Stern-Gerlach experiment contains the two kinds of K-T operators, the one, $\exp \left(i \frac{\sigma_{z}}{2} \otimes \hat{\theta}\right)$, to couple the quantum observable $\boldsymbol{\sigma}$ with the 
angle variable $\hat{\theta}=2 t \mu B_{z} / \hbar$ and the other one, $\exp \left(\frac{i}{\hbar} \hat{p}_{z} \otimes \hat{z}\right)$, corresponding to the translations $z \rightarrow z+a$ of $z$ due to the $z$-dependence of $B_{z}(\mathbf{x}) \simeq B_{0}+B_{1} z$, the latter of which is responsible for the direct amplification of the former coupling. This explains a dynamical mechanism to transcribe the information on the spin direction into the momentum change in the orbital motion of the electron, which allows us to achieve the quantitative estimation as shown above.

Aside from the Stern-Gerlach case, a unitary coupling of the similar nature has been found in [18]2. Our focus here is, however, to clarify the universal essence of such couplings via external fields, which seems impossible without the use of K-T operators.

\subsection{Adiabatic perturbation and adiabaticity condition}

In the above discussion for deriving the momentum change of the electron, we neglected such secondary effects as the terms come from $B_{x}$ or $\frac{\partial B_{x}}{\partial z}$. Since these effects are outside the scope of the above ideal situation of amplification, we need to estimate them as correction terms in the next step. Without the necessity to develop the general method for treating these secondary terms, we already know some of typical methodology for these estimation; in some cases (including the Stern-Gerlach case) it would be called 'adiabaticity conditions'. For Stern-Gerlach experiment, this condition can be interpreted as the one under which the effect of spin-flips caused by the factor $\frac{\partial B_{x}}{\partial z}$ remains small enough compared with that of $B_{z}$. In this section, we confirm that the adiabaticity condition surely gives the consistency in the present context by an elementary discussion.

'Adiabatic perturbation' originally means a coupling of a quantum system with an external force which changes the system slowly enough in comparison to the typical time scales of intrinsic transitions among quantum states but whose changes along the direction of condensed order parameters can eventually accumulate into a visible size. The general essence of the adiabaticity can be formulated in such a condition as $\left|U_{f i}\right| \ll 1$, in terms of the rate of change of the matrix elements of Hamiltonian $H$ defined by

$$
U_{f i}:=\frac{\hbar}{\left(E_{f}-E_{i}\right)^{2}}\left(\frac{\partial H}{\partial t}\right)_{f i}
$$

between the initial and final states with the energies $E_{i}$ and $E_{f}$, respectively. The physical meaning of the quantity $U_{f i}$ can be understood by the following

\footnotetext{
${ }^{2}$ The paper by Prof. G. Emch has been brought to our attention by Prof. Ohya, to whom we express our gratitudes.
} 
reformulation of it:

$$
U_{f i}=\frac{(\Delta H)_{f i}}{(\Delta E)_{f i}}=\frac{\left(\frac{\partial H}{\partial t} \Delta t\right)_{f i}}{E_{f}-E_{i}}=\frac{\left(\frac{\partial H}{\partial t}\right)_{f i} \omega_{f i}^{-1}}{E_{f}-E_{i}},
$$

with $\Delta t:=\frac{\hbar}{E_{f}-E_{i}}=: \omega_{f i}^{-1}$ which sets up the standard time scale for the comparison. The requirement $\left|U_{f i}\right| \ll 1$ can now be understood as the selfconsistency condition for a process to change the values of the order parameters describing a given inter-sectorial structure of the quantum-classical composite system, without destroying the whole sector structure: if the change rate $\frac{\partial H}{\partial t}$ of the Hamiltonian is very small, it should be almost perpendicular to the main 'tangential direction' of the changes caused by the external force in favour of the change in the order parameters. Therefore, $\Delta t$ can be interpreted as the 'almost intrinsic' time scale of the microscopic motions of the intra-sectorial quantum system put in a background with slowly changing order parameters, in which $\omega_{f i}$ can represent, for instance, the frequency of the light emitted in the transition. Then the numerator in $U_{f i}=\frac{(\Delta H)_{f i}}{(\Delta E)_{f i}}$ is the change $(\Delta H)_{f i}=\left(\frac{\partial H}{\partial t} \Delta t\right)_{f i}$ of the matrix element of $H$ from the initial $i$ to final states $f$ caused by the adiabatic perturbation during the time interval $\Delta t$, which is to be compared with the denominator $(\Delta E)_{f i}=E_{f}-E_{i}$ given by the energy difference almost intrinsic to the quantum system.

Going back to the Stern-Gerlach case, the interaction Hamiltonian is given by

$$
\widetilde{H_{I}}=\frac{e \hbar}{2 \mu_{0} m c} \mathbf{B} \cdot \boldsymbol{\sigma}=\frac{e \hbar}{2 \mu_{0} m c}\left(B_{x} \sigma_{x}+B_{z} \sigma_{z}\right),
$$

The decomposition of the external magnetic field into its $z$-component $B_{z}$ and the remaining $B_{x}$ can be understood as the one into the directions to preserve and to disturb the sector structure according to the eigenvalues of $\sigma_{z}$. Therefore, the dominant term in this Hamiltonian to disturb the spin direction due to the spin-flips is identified with

$$
H_{I}=\frac{e \hbar}{2 \mu_{0} m c} B_{x} \sigma_{x}
$$

The size of the effect due to this term should be estimated to preserve the visibility aspect due to $B_{z}$.

As each trajectory of electron can be considered as a smooth curve in $\mathbb{R}^{3}$ parameterized by the time parameter $t$, the time derivative of $H_{I}$ is calculated as

$$
\frac{\partial H_{I}}{\partial t}=\frac{e \hbar}{2 \mu_{0} m c} \frac{d x}{d t} \frac{\partial B_{x}}{\partial x} \sigma_{x}
$$


Here we introduce an approximation $B_{x} \simeq B_{x}(z=0)+\frac{\partial B_{x}}{\partial z} z$. In terms of a basis of eigenstates of $\sigma_{z}$, we can estimate and obtain a representation of off-diagonal matrix elements

$$
\left(\frac{\partial H_{I}}{\partial t}\right)_{f i}=\frac{e \hbar}{2 \mu_{0} m c} v z \frac{\partial^{2} B_{x}}{\partial x \partial z} \int \overline{\psi_{f}(x)} \sigma_{x} \psi_{i}(x) d x
$$

under the assumption that the velocity $d x / d t$ of the electron can be replaced by the typical velocity $v$ of thermal electrons. Owing to the first condition for $\partial H / \partial t$ to be adiabatic, the derivative of the external magnetic field can be approximated in the context of the estimate by $\partial B_{x} / \partial z \sim B_{z} /(\Delta x)$, where $\Delta x$ represents the range in which the magnetic field exists.

In terms of the Larmor frequency of the thermal electron $\omega=e B_{z} / 2 \mu_{0} m c$, the changing rate in which we are interested is essentially given by

$$
\begin{aligned}
U_{f i} & =\frac{v z \frac{\partial}{\partial x}\left(\frac{B_{z}}{\Delta x}\right)}{\omega B_{z}} \\
& =v \frac{z}{\omega \Delta x} \frac{1}{B_{z}} \frac{\partial B_{x}}{\partial z} .
\end{aligned}
$$

in the use of the rotation-free condition $\frac{\partial B_{z}}{\partial x}=\frac{\partial B_{x}}{\partial z}$ of the magnetic field B. Thus the adiabaticity condition $\left|U_{f i}\right| \ll 1$ can be written down as

$$
\frac{\partial B_{x}}{\partial z} \ll \frac{\omega}{v} B_{z} .
$$

This inequality is nothing but the condition imposed on the arrangement of external magnetic field in order to guarantee the ideal amplification of spin variables.

\section{Summary}

In this paper we have formulated a unified scheme of measurement and amplification processes based on the notion of Micro-Macro duality. In this context, the duality relation (or, in more general contexts, adjunction) between $\mathcal{M}$ as the microscopic system and $\operatorname{Spec}(\mathcal{A})$ as the macroscopic observational data controlled by the K-T operator has played the essential role, on the basis of which we have obtained a clear understanding of how microscopic states are amplified into macroscopic level as discussed in $\oint 3$. We hope that this essence of amplification processes will shed some new lights on various problems involving different scales or levels (especially, 'Micro' and 'Macro') such as the coexistence of different phases and their boundaries, the problem of emergence of macroscopic structures from microscopic worlds, and so on. 


\section{Acknowledgments}

One of the authors (I. O.) would like to express his sincere thanks to Prof. M. Ohya, Prof. L. Accardi and Prof. T. Hida for their encouragements. Both of the authors are very grateful to Mr. H. Ando, Mr. T. Hasebe and Mr. H. Saigo for their valuable discussions in the early stage of the work.

\section{References}

[1] Ojima, I., A unified scheme for generalized sectors based on selection criteria - Order parameters of symmetries and of thermality and physical meanings of adjunctions -, Open Systems and Information Dynamics, 10 (2003), 235-279.

[2] Ojima, I., Micro-macro duality in quantum physics, pp.143-161 in Proc. Intern. Conf. on Stochastic Analysis, Classical and Quantum, World Scientific (2005).

[3] Ojima, I. and Takeori, M, How to observe quantum fields and recover them from observational data? - Takesaki duality as a Micro-Macro duality -, Open Systems and Information Dynamics, 14, 307-318 (2007) (math-ph/0604054).

[4] Dixmier, J., $C^{*}$-Algebras, North-Holland (1977); Pedersen, G., $C^{*}$ Algebras and Their Automorphism Groups, Academic Press (1979).

[5] Ozawa, M., Quantum measuring processes of continuous observables. J. Math. Phys. 25, 79-87 (1984); Publ. RIMS, Kyoto Univ. 21, 279-295 (1985); Ann. Phys. (N.Y.) 259, 121-137 (1997).

[6] Tatsuuma, N., A duality theory for locally compact groups, J. Math. Kyoto Univ. 6 (1967), 187-217; Takesaki, M., A characterization of group algebras as a converse of Tannaka-Stinespring-Tatsuuma duality theorem, Amer. J. Math. 91 (1969), 529-564.

[7] Enock, M. and Schwartz, J-M., Kac Algebras and Duality of Locally Compact Groups, Springer-Verlag (1992).

[8] Baaj, S. and Skandalis, G., Unitaires multiplicatifs et dualité pour les products croisés de $\mathrm{C}^{*}$-algèbres, Ann. scient. Éc. Norm. Sup., $4^{\circ}$ série, 26 (1993), 425-488.

[9] Ozawa, M., Perfect correlations between noncommuting observables, Phys. Lett. A, 335, 11-19 (2005).

[10] The earlier version of the mathematical formulation of amplification processes proposed by one of the authors (I.O.) can be found in the 
following articles: Ojima, I., Lévy process and innovation theory in the context of Micro-Macro duality, A brief summary of talks at the 5th Lévy Seminar (2006), edited by T. Hida; Ojima, I., Micro-Macro duality and emergence of macroscopic levels, Quantum Probability and White Noise Analysis, 21, 217-228 (2008) (math-ph/07052945).

[11] Accardi, L., Noncommutative Markov chains, in Intern. School of Math. Phys., Camerino, pp. 268-295 (1974); Topics in quantum probability, Phys. Rep., 77 (1981) 169-192.

[12] Ojima, I. and Ozawa, M., Unitary representations of the hyperfinite Heisenberg group and the logical extension methods in physics, Open Systems and Information Dynamics 2, 107-128 (1993).

[13] Ojima, I. and Tanaka, S., State preparation, wave packet reduction and repeated measurements (in Japanese), Part III, Chapater 2, pp. 235243 in Quantum Information and Evolution Dynamics, ed. by Ohya, M. and Ojima, I., Makino-Shoten (1996).

[14] Bohm, D., Quantum Theory, New York: Prentice Hall, pp. 326-327, 501-505 (1951).

[15] Tomonaga, S., Quantum Mechanics (in Japanese), Vols. I and II, Misuzu-Shobou (2nd. ed. 1969).

[16] See pp. 225-226 in Ojima, I., Micro-Macro duality and emergence of macroscopic levels, Quantum Probability and White Noise Analysis, 21, 217-228 (2008) (math-ph/07052945).

[17] Helgason, S., The Radon Transform, Birkhäuser (2nd. ed. 1999).

[18] Emch, G., On quantum measurement processes, Helv. Phys. Acta 45 (1972/73) 1049-1056. 